\title{
EFECTO DE LA COMBINACIÓN DE GLUCOSA OXIDASA/ GLUCOSA SOBRE EL CRECIMIENTO DE BACTERIAS DEL GÉNERO Salmonella AISLADAS DE AVES DE CORRAL
}

\section{EFFECT OF THE GLUCOSE OXIDASE /GLUCOSE COMBINA- TION ON THE GROWTH OF THE GENUS Salmonella BACTE- RIA ISOLATED FROM POULTRY}

\author{
Nirza C. Noguera-Machado ${ }^{1}$, Luis E. Ojeda-Ojeda ${ }^{2}$, Luis Pérez-Ybarra ${ }^{3}$, Mariela Brett ${ }^{4}$, \\ Keilyn García ${ }^{5}$. Antonio Yépez ${ }^{6}$, Juana L. Triana ${ }^{7}$
}

\begin{abstract}
${ }^{1}$ Ingeniero Agrónomo, Doctora en Ciencias, Profesora Titular de la Universidad de Carabobo. Jefa de la Sección de Biotecnología Agroalimentaria del Instituto de Investigaciones Biomédicas "Dr. Francisco J. Triana Alonso" UC. Maracay, Estado Aragua, Venezuela, e-mail: nirza.noguera@gmail.com; '2icenciado en Química, Magíster en Ciencia y Tecnología de Alimentos, Profesor Titular de la Universidad de Carabobo e Investigador de la Sección de Biotecnología Agroalimentaria del Instituto de Investigaciones Biomédicas "Dr. Francisco J. Triana Alonso" UC. Maracay, Estado Aragua, Venezuela, e-mail: lojeda2@ uc.edu.ve; ${ }^{3}$ Ingeniero Agrónomo, Magíster en Estadística. Profesor Titular de la Universidad de Carabobo e Investigador de la Sección de Biotecnología Agroalimentaria del Instituto de Investigaciones Biomédicas "Dr. Francisco J. Triana Alonso" UC. Maracay, Estado Aragua, Venezuela, e-mail: Imperez4@uc.edu.ve; ${ }^{4}$ Médico Veterinario, Magíster en Microbiología, Investigadora del Laboratorio de Patología Aviar del Instituto Nacional de Investigaciones Agrícolas (INIA), Estado Aragua, Venezuela, e-mail: marielabrett@gmail.com; ${ }^{5}$ Médico Veterinario, Magíster en Microbiología, Investigadora del Laboratorio de Patología Aviar del Instituto Nacional de Investigaciones Agrícolas (INIA), Estado Aragua, Venezuela, e-mail: carole2582@gmail.com; ${ }^{6}$ Técnico, Personal administrativo asociado al Instituto de Investigaciones Biomédicas "Dr. Francisco J. Triana Alonso" UC. Maracay, Estado Aragua, Venezuela; ${ }^{7}$ Licenciada en Química, Magíster en Bioquímica y Biología Molecular, Profesora Titular de la Universidad de Carabobo y Jefa de la Sección de Proteínas y Ácidos Nucleicos del Instituto de Investigaciones Biomédicas "Dr. Francisco J. Triana Alonso" UC. Maracay, Estado Aragua, Venezuela, e-mail: lediatriana@yahoo.es
\end{abstract}

\section{Rev. U.D.C.A Act. \& Div.Cient.21(1): 127-136, Enero-Junio, 2018 https://doi.org/10.31910/rudca.v21.n1.2018.671}

\section{RESUMEN}

En la industria avícola, las infecciones por Salmonella son un problema, debido a los efectos sobre la producción y los riesgos a la salud pública. En las aves infectadas puede ocurrir una diseminación sistémica de las bacterias, que afecta los órganos y favorece, tanto la transmisión entre las aves como la contaminación de los productos avícolas, tales como las carnes, durante el proceso de sacrificio de los animales. Se decidió evaluar el efecto que diferentes combinaciones de glucosa oxidasa (GOX) y glucosa tienen sobre el crecimiento de diversos serotipos de Salmonella aislados de aves en crecimiento y de engorde, y determinar su potencial como antibacteriano. Se tomaron muestras de corazón y de ciego de aves de 7 y 42 días de edad y se aislaron e identificaron S. typhimurium y S. enteritidis. Estas bacterias fueron cultivadas en medio Luria-Bertani (LB) en presencia de GOX $(0,5 ; 1$ y $2 U / \mathrm{mL})$ y glucosa $(0,5,1$ y $2 \%)$, en combinaciones bajo un diseño factorial $3^{2}$. El crecimiento fue monitoreado por el cambio de absorbancia a $600 \mathrm{~nm}$, durante 6 horas de incubación. Se observó una reducción significativa del crecimiento de ambos serotipos al utilizar $2 U / \mathrm{mL}$ de GOX y diferentes concentraciones de glucosa, lo cual, demostró la capacidad antibacteriana que posee el sistema GOX/glucosa sobre este género, por lo que se podría emplear como un aditivo para la conservación de carnes de aves y productos derivados, a fin de alargar su vida de anaquel y minimizar riesgos a la salud.

Palabras clave: Pollos, S. typhimurium, S. enteritidis, enzima antibacteriana, conservante natural.

\section{SUMMARY}

In the poultry industry, Salmonella infections are a problem due to the effects on production and risks to public health. In infected birds, a systemic spread of bacteria can occur, which affects the organs and favors both the transmission between birds and the contamination of poultry products, such as meat, during the animal's slaughter process. It was evaluated the effect that different combinations of glucose oxidase (GOX) and glucose have on the growth of different 
Salmonella serotypes isolated from broilers, and thus determine their potential as antibacterial. Heart and caecal samples were taken from birds 7 and 42 days old. S. typhimurium and $S$. enteritidis were isolated and identified. These bacteria were cultured in Luria-Bertani medium (LB) in the presence of GOX $(0.5,1$ and $2 \mathrm{U} / \mathrm{mL})$ and glucose $(0.5,1$ and $2 \%$ ) in combinations under a factorial design $3^{2}$. Growth was monitored by absorbance change at $600 \mathrm{~nm}$ for 6 hours of incubation. A significant reduction of the growth of both serotypes was observed when using $2 \mathrm{U} / \mathrm{mL}$ of GOX and different concentrations of glucose. This demonstrated the antibacterial capacity that the GOX/glucose system has on this genus, so it could be used as an additive for the preservation of poultry meats and derived products, in order to lengthen its storage time and minimize health risks.

Key words: Chickens, S. typhimurium, S. enteritidis, antibacterial enzyme, natural preservative.

\section{INTRODUCCIÓN}

El género Salmonella figura entre los patógenos más comúnmente asociados a las enfermedades de transmisión alimentaria, que afecta a millones de personas cada año. Se estima que, anualmente en el mundo, se enferman unos 600 millones de personas por ingerir alimentos contaminados y, alrededor de 420.000, mueren por esta misma causa (WHO, 2015). La infección gastrointestinal ocasionada por la bacteria Salmonella, denominada salmonelosis, es una de las enfermedades de transmisión alimentaria más comunes y ampliamente extendidas en todo el mundo afectando, tanto a países en desarrollo como a los desarrollados (Paniagua-Contreras et al. 2008). Se caracteriza por la aparición brusca de fiebre, dolor abdominal, diarrea, náuseas y, ocasionalmente, vómitos. Los síntomas de la enfermedad comienzan a manifestarse entre 6 y 72 horas después de la ingesta de alimentos o aguas contaminadas y tiene una duración entre 2 y 7 días. En la mayoría de los casos, los síntomas por salmonelosis son relativamente leves y los pacientes se recuperan sin tratamiento específico; sin embargo, en algunos casos, particularmente en niños pequeños, ancianos y personas con enfermedades crónicas, la deshidratación causada por la enfermedad puede ser grave y poner en riesgo su vida (WHO, 2016).

Salmonella es un género de bacilos gramnegativos, no esporulados, anaerobios facultativos, que pertenece a la familia Enterobacteriaceae (WHO, 2016). Su nomenclatura suele ser compleja y variante, debido al concepto de serotipos o serovares introducido por Kauffmann (1966), el cual, no tiene el nivel taxonómico de las especies y no cumple con el Código Internacional de Nomenclatura Bacteriana. El Centro de Referencia e Investigación de Salmonella de la Organización Mundial de la Salud en el Instituto Pasteur (WHO Colla- borating Centre for Reference and Research on Salmonella), ha clasificado el género en dos especies, a saber: Salmonella bongori y Salmonella entérica, esta última, dividida en 6 subespecies, que se diferencian por sus características bioquímicas y genéticas. Cada subespecie, a su vez, contiene serovariedades o serotipos, definidas por su fórmula antigénica (Uribe \& Suárez, 2006). Los dos serotipos más comunes asociados a gastroenteritis en humanos son S. enteriditis y $S$. typhimurium, que pertenecen a la especie $S$. enterica subespecie enterica (Uribe \& Suárez, 2006; Paniagua-Contreras et al. 2008; Rincón et al. 2011).

Entre los alimentos en los que comúnmente se han aislado estas bacterias están las carnes de aves de corral y de cerdos, los huevos y los productos lácteos (Camacho et al. 2010; Molina et al. 2010; Rincón et al. 2011), también se ha reportado su presencia en vegetales (Nillian et al. 2011). Los productos avícolas, en general, son considerados alimentos de alto riesgo, ya que sus características fisicoquímicas favorecen la proliferación de microorganismos. Uno de los procesos críticos donde ocurre con mayor frecuencia la contaminación es durante el sacrificio o beneficio, especialmente, en la etapa de evisceración, ya que se liberan bacterias existentes en el sistema gastrointestinal (Molina et al. 2010; Mercado et al. 2012).

La contaminación de la carne de aves se considera indeseable, pero inevitable. En este sentido, Mercado et al. (2012) llevaron a cabo una revisión y encontraron que, en los diferentes estudios analizados, las canales de las aves estaban contaminadas con microorganismos. El número y la especie de éstos variaba, con una alta prevalencia de Salmonella y una carga inicial, que podía ser baja al principio del proceso de beneficio, de acuerdo con las condiciones sanitarias de crianza de los animales, pero se suele incrementar en función de la manipulación, el procesamiento, el tiempo y la temperatura de almacenamiento.

Esta problemática ha generado búsqueda de medidas, que permitan reducir la contaminación, desde normas sanitarias, con puntos de control, para la limpieza y la desinfección en las distintas etapas del proceso productivo, hasta la aplicación de biotecnologías dirigidas a la conservación y a la preservación de las carnes y sus derivados, como el uso de las enzimas glucosa oxidasa y catalasa (Fresl et al. 1984), bacterias ácido láctica y bacteriocinas (Vásquez et al. 2009; Beristain-Bauza et al. 2012; Castañeda et al. 2013) y aditivos naturales comestibles (Graciano-Cristóbal et al. 2017).

En cuanto al uso de las enzimas, aunque Frels et al. (1984) no observaron una inhibición significativa del crecimiento de las bacterias del género Salmonella, encontraron que durante los primeros 6 días de almacenamiento, el contaje de las bacterias resultó menor en las muestras tratadas con 
las enzimas con respecto al control. Investigaciones relacionadas con la enzima glucosa oxidasa (EC 1.1.3.4), han demostrado su efecto antibacteriano sobre diferentes especies, como Escherichia coli enterotóxica PM 015, Salmonella Derby BP 177 (Massa et al. 2001), Pseudomonas fragi (Yoo \& Rand,1995) y Escherichia coli ATCC 25922 (Noguera et al. 2014).

En consecuencia, en el presente trabajo, se evaluó el efecto que diferentes combinaciones de glucosa oxidasa (GOX) y glucosa tienen sobre el crecimiento de dos serotipos de Salmonella aisladas de aves provenientes de granjas del estado Aragua, Venezuela, a fin de determinar su potencial como aditivos, para mejorar la calidad microbiológica de este tipo de alimentos y sus derivados.

\section{MATERIALES Y MÉTODOS}

Aislamiento e identificación de bacterias. Se tomaron muestras de órganos de aves de engorde con 7 y 42 días de edad, procedentes de granjas del estado Aragua, Venezuela. Específicamente, se trabajó con corazón de aves en crecimiento (7 días) y ciegos de aves de engorde (42 días), en el Laboratorio de Patología Aviar del Instituto Nacional de Investigaciones Agrícolas (INIA) del estado Aragua, Venezuela.

El procedimiento, se hizo bajo la Norma 1291-88 (COVENIN) y de acuerdo con lo previsto por los estándares internacionales (Watman et al. 2008). Se realizó la necropsia de órganos, para evidenciar lesiones macroscópicas compatibles con Salmonella y se tomaron muestras de corazón y de ciego. Las mismas fueron homogenizadas e incubadas por separado, en caldo de enriquecimiento Tetrationato, a $42^{\circ} \mathrm{C}$, por 24 horas. Posteriormente, se sembraron en agar selectivo XLT4 y verde brillante, a $37^{\circ} \mathrm{C}$, por $24-48$ horas. Por cada medio selectivo, se seleccionaron las colonias típicas o sospechosas de Salmonella spp. y se sometieron individualmente a una batería inicial de pruebas bioquímicas. La identificación serológica, se procedió mediante ensayos de aglutinación con antisueros Poly A y monovalentes, marca Difco.
Extracto enzimático y sustrato. Se trabajó con un extracto enzimático de GOX con 8,14U/mL, producido en el laboratorio de Biotecnología Agroindustrial del Instituto de Investigaciones Biomédicas "Dr. Francisco Triana-Alonso" (BIOMED-UC), a partir de Aspergillus niger (aislado clínico), de acuerdo con la metodología establecida por Zoghbi et al. (2008). Las unidades de actividad enzimática del extracto, se determinaron por el método colorimétrico, descrito por Trinder y modificado por Zoghbi et al. (2008). Como sustrato, se utilizó glucosa marca Sigma $^{\circledR}$ especificaciones G-8720.

Efecto del sistema GOX/glucosa sobre los serotipos de Salmonella aislados. Se diseñó un experimento bajo un arreglo factorial de tratamientos $3^{2}$, para evaluar el efecto que diferentes combinaciones de GOX y glucosa pueden tener el crecimiento de las cepas de Salmonella, aisladas previamente. Como medio de cultivo, se utilizó el Luria-Bertani (LB), por ser rico en nutrientes, de fácil elaboración y, en el cual, crece una amplia gama de cepas pertenecientes a las Enterobacterias (Garboza et al. 2011). Los niveles o concentraciones utilizados de GOX fueron $0,5,1$ y $2 \mathrm{U} / \mathrm{mL}$ y los de glucosa $0,5,1$ y $2 \%$, generándose un total de 9 tratamientos (Tabla 1), más la adición de un control correspondiente al medio LB sin aditivos. Estos valores, se establecieron en función de investigaciones previas, en las que se demostró un efecto antibacteriano del sistema, sobre diferentes cepas cultivadas en distintos medios (Massa et al. 2001; Noguera et al. 2014). Todos los cultivos, se realizaron en un volumen final de $25 \mathrm{~mL}$ de medio (unidad experimental). Se establecieron tres réplicas de cada tratamiento y una sola unidad experimental por réplica, a partir, de las cuales, se generaron seis observaciones repetidas en el tiempo.

El procedimiento, se inició con un pre-inóculo de las bacterias aisladas cultivadas en medio LB, incubadas a $37^{\circ} \mathrm{C}$, por 12 horas. Se tomaron $2 \mathrm{~mL}$ del pre-inóculo (equivalente a 0,1 Abs600nm de turbidez inicial) y se cultivaron en los distintos tratamientos establecidos. La incubación, se realizó a $37^{\circ} \mathrm{C}$, bajo agitación continua, a 200rpm, en un incubador marca New Brunswick Scientific, Controlled Environment Incubator Shaker (New Brunswick Scientific Co., Inc., Edison, New

Tabla 1. Tratamientos generados por la combinación de GOX y glucosa, presentes en el medio de cultivo (LB), bajo un diseño factorial $3^{2}$.

\begin{tabular}{|c|c|c|c|}
\cline { 2 - 4 } \multicolumn{1}{c|}{} & \multicolumn{3}{c|}{ Concentración de Glucosa (\%) } \\
\hline Concentración de GOX $(\mathbf{U} / \mathbf{m L})$ & $\mathbf{0 , 5}$ & $\mathbf{1}$ & $\mathbf{2}$ \\
\hline $\mathbf{0 , 5}$ & $\mathrm{T} 1$ & $\mathrm{~T} 2$ & $\mathrm{~T} 3$ \\
\hline $\mathbf{1}$ & $\mathrm{T} 4$ & $\mathrm{~T} 5$ & T6 \\
\hline $\mathbf{2}$ & $\mathrm{T} 7$ & $\mathrm{~T} 8$ & T9 \\
\hline
\end{tabular}


Jersey, (USA). El crecimiento fue monitoreado por turbidimetría, midiendo la absorbancia a 600nm (Abs600nm), para los tiempos de 0,60, 180, 240, 300 y 360 minutos. Las lecturas absorbancia, se efectuaron en un espectofotómetro marca BECKMAN, modelo DU® 650 (Beckman Instruments, Inc., Fullerton, CA, USA). La respuesta analizada fue la diferencia de absorbancia en los tiempos desde los 60 minutos hasta los 360 minutos con respecto a la absorbancia inicial, a los 0 minutos, es decir, sea Abst600nm, la absorbancia de una muestra cualesquiera en el instante t, y Abso 600nm, la absorbancia para el tiempo 0; el dato analizado fue $\Delta \mathrm{Abst} 600 \mathrm{~nm}$, que viene dado por:

$$
\Delta \text { Abst } 600 \mathrm{~nm}=\text { Abst } 600 \mathrm{~nm}-\text { Abs0 } 600 \mathrm{~nm}
$$

Análisis Estadístico. Se realizaron tres tipos de análisis. El primero, un análisis de varianza (ANOVA), con observaciones repetidas para el arreglo factorial de tratamientos $3^{2}$, a través del tiempo. Esto, para determinar si el crecimiento de los serotipos aislados fue condicionado por las distintas combinaciones de GOX y glucosa establecidas. Para ello, primero se aplicó la prueba de Mauchly, con el fin verificar si la matriz de varianza-covarianzas resultaba esférica o no y si requería o no el cálculo de p-valores ajustados. Las pruebas de medias para los efectos principales, se llevaron a cabo utilizando la prueba de comparaciones múltiples de Tukey. El segundo tipo de análisis, se efectuó considerando los nueve tratamientos del arreglo factorial más el control establecido y se aplicó el ANOVA de una vía y la prueba de comparaciones de tratamiento contra control de Dunnett, en cada instante medido, con el fin de identificar cuáles combinaciones de GOX y glucosa, presente en el medio, ocasionaban un menor crecimiento de las bacterias aisladas con respecto al crecimiento de las mismas bacterias en el medio sin aditivos (control). Por último, se aplicaron pruebas de comparaciones múltiples, utilizando la prueba de Scheffé para contrastes ortogonales, a fin de establecer la combinación de GOX y glucosa con mejor efecto inhibitorio, sobre el crecimiento de cada bacteria, durante el tiempo del experimento.

Se trabajó al nivel de significación de $5 \%$, por lo cual, un resultado se consideró estadísticamente significativo, siempre que $p \leq 0,05$. Los datos, se analizaron con los programas estadísticos SPSS 21.0 (ANOVA con observaciones repetidas en el tiempo y prueba de comparaciones múltiples de Tukey) y Statistix 9.0 (ANOVA de una vía en cada instante medido durante el ensayo, prueba de Dunnett y prueba para contrastes ortogonales de Scheffé).

\section{RESULTADOS Y DISCUSIÓN}

Los serotipos aislados e identificados de las muestras analizadas se correspondieron con S. entérica, subespecie enterica serovariedad Typhimurium (S. typhimurium) y S. entérica, subespecie enterica serovariedad Enteritidis (S. enteritidis). A continuación, se describen los efectos que la adición al medio de cultivo de la enzima GOX, en combinación con la glucosa, tienen sobre el crecimiento de estas bacterias.

\section{Efecto del sistema GOX/glucosa sobre el crecimiento de los serotipos aislados.}

\section{A) S. typhimurium.}

En la figura $1 \mathrm{~A}$, se puede observar que el crecimiento de este serotipo tuvo un comportamiento distinto de acuerdo con la combinación de GOX y glucosa utilizadas, tal como lo demuestra el análisis estadístico.

El ANOVA para el arreglo factorial $3^{2} \operatorname{con} p$-valores ajustados demostró que hubo un efecto, tanto de la concentración de enzima ( $F=1595 ; 2$ gdl; $p<0,001)$ como de la concentración de glucosa $(F=100,77 ; 2$ gdl; $p<0,001)$, sobre el crecimiento bacteriano y que, además, hubo una interacción significativa entre estos factores ( $F=22,38 ; 4 \mathrm{gdl} ; \mathrm{p}<0,001)$. Tal como era de esperarse, dada la naturaleza de la reacción bioquímica, la magnitud del efecto inhibidor depende, tanto de la concentración de enzimática como del sustrato (Tiina \& Sandholm, 1989; Holzapfel et al. 1995). Este análisis, también confirmó un efecto significativo del factor tiempo $(\mathrm{F}=2088 ; 5 \mathrm{gdl} ; \mathrm{p}<0,001)$ y de sus distintas interacciones GOX-tiempo $(\mathrm{F}=328,73$; 10gdl; $\mathrm{p}<0,001)$, glucosa-tiempo $(\mathrm{F}=26,82 ; 10 \mathrm{gdl} ; \mathrm{p}<0,001)$ y GOX-glucosa-tiempo $(F=10,51 ; 20 \mathrm{gdl} ; \mathrm{p}<0,001)$.

Respecto a los efectos principales, la prueba de comparaciones múltiples de Tukey indicó que los promedios de los niveles de GOX y glucosa fueron diferentes, de tal forma que el mayor crecimiento de $S$. typhimurium ocurrió con el nivel más bajo de GOX $0,5 \mathrm{U} / \mathrm{mL}\left(=1,009^{\mathrm{A}}\right)$, seguido de $1 \mathrm{U} /$ $\mathrm{mL}\left(=0,681^{\mathrm{B}}\right) \mathrm{y}$, el menor crecimiento de esta bacteria, se obtuvo con la adición de $2 \mathrm{U} / \mathrm{mL}$ de GOX $\left(=0,153^{\mathrm{C}}\right)$. Para la glucosa, también se observó que el mayor crecimiento ocurrió al nivel más bajo, $0,5 \%\left(=0,726^{A}\right)$, seguido de $1 \%$ $\left(=0,607^{\mathrm{B}}\right)$ y menor crecimiento, al $2 \%\left(=0,509^{\mathrm{C}}\right)$. Las letras en los superíndices representan los grupos de medias de Tukey con diferencias significativas. Asimismo, el efecto inhibitorio, ocasionado por los niveles del factor GOX, resultó ser de mayor magnitud que el generado por la glucosa.

La interacción GOX-glucosa, promediada a lo largo del tiempo, ocurrió a diferentes tasas. La tasa más alta, se obtuvo para los niveles 0,5 y $1 \mathrm{U} / \mathrm{mL}$ y la menor, para $2 \mathrm{U} / \mathrm{mL}$ de GOX (Figura 2A), es decir, que tal como lo afirmaron Tiina \& Sandholm (1989), bajas concentraciones de enzima requieren mayor concentración de sustrato para ocasionar una reducción significativa en el crecimiento bacteriano.

La interacción GOX-tiempo, promediada sobre los niveles de glucosa, fue homogénea en los primeros 60 minutos; a partir 

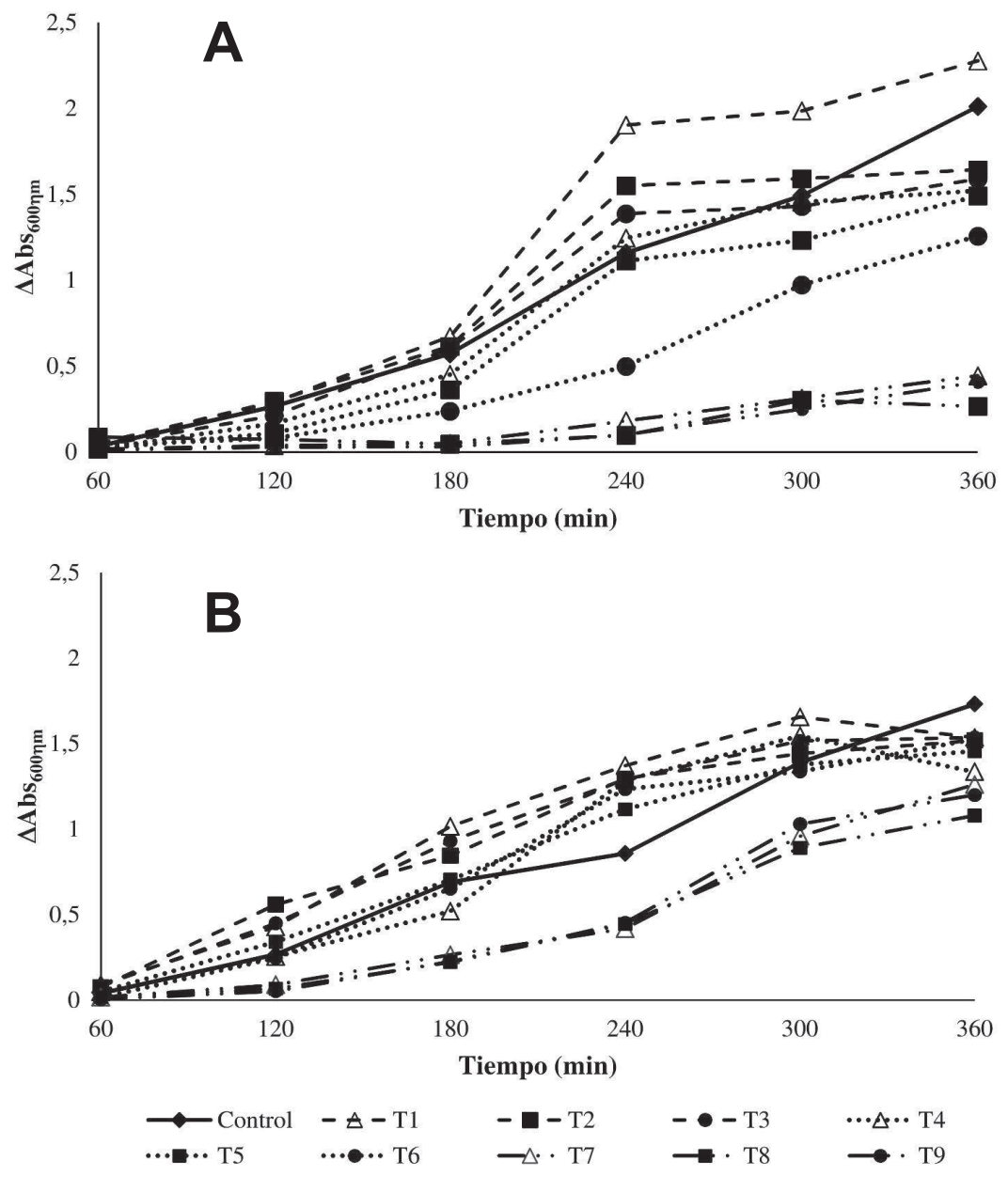

Figura 1. Curvas de crecimiento de las cepas de Salmonella aisladas y cultivadas en medio LB, con diferentes combinaciones de GOX y glucosa: (A) S. typhimurium, (B) S. enteritidis.

Control: medio LB sin aditivos; T1: medio LB + GOX0,5/glucosa0,5; T2: medio LB + GOX0,5/glucosa1,0; T3: medio LB + GOX0,5/glucosa2,0; T4: medio LB + GOX1,0/glucosa0,5; T5: medio LB + GOX1,0/glucosa1,0; T6: medio LB + GOX 1,0/ glucosa2,0; T7: medio LB + GOX2,0/ glucosa0,5; T8: medio LB + GOX2,0/glucosa1,0; T9: medio LB + GOX2,0/glucosa 2,0.

de los 120 minutos, el crecimiento de S. typhimurium fue menor, en la medida que incrementó la concentración de la enzima, de tal forma, que las menores respuestas de $\Delta$ Abs$600 \mathrm{~nm}$ se lograron al utilizar $2 \mathrm{U} / \mathrm{mL}$ de GOX (Figura 2B).

La interacción glucosa-tiempo, promediada sobre los niveles de GOX, exhibió un comportamiento similar a la interacción GOX-tiempo; el menor crecimiento, se presentó con la glucosa al 2\%, seguido de la concentración de glucosa $1 \%$ (Figura 2C).

En cuanto a la comparación de las combinaciones de GOX y glucosa con respecto al control, los resultados del ANOVA y la prueba de comparación de Dunnett, demostraron que en la primera hora no hubo diferencias; a partir de la segunda hora (120 minutos) hubo diferencias significativas y los tratamientos T7, T8 y T9 mantuvieron sus $\Delta$ Abs600nm por debajo del control, hasta el final del experimento. Implica que, pro- bablemente, el efecto inhibitorio lo ocasionó la acumulación en el medio de cultivo de los productos de reacción, el peróxido de hidrógeno y el ácido glucónico, los cuales, son los responsables de la toxicidad del sistema para las células bacterianas (Holzapfel et al. 1995; Bankar et al. 2009). Específicamente, el peróxido de hidrógeno ocasiona daños a nivel de los grupos sulfidrilos de la membrana celular, afectando el sistema de transporte de la glucosa, por inactivación de las enzimas hexoquinasa y la gliceraldeido 3-fosfato deshidrogenasa. Los radicales libres del ion superóxido y el hidroxilo también pueden ocasionar daños en la célula bacteriana, a nivel del ADN (Yang, 2000). El efecto inhibitorio del ácido glucónico está asociado con la reducción del pH del medio (Massa et al. 2001).

La velocidad con la que se generan estos productos depende de la concentración de la enzima y del sustrato, por lo que, una alta dosis de GOX, favorece una rápida producción de 

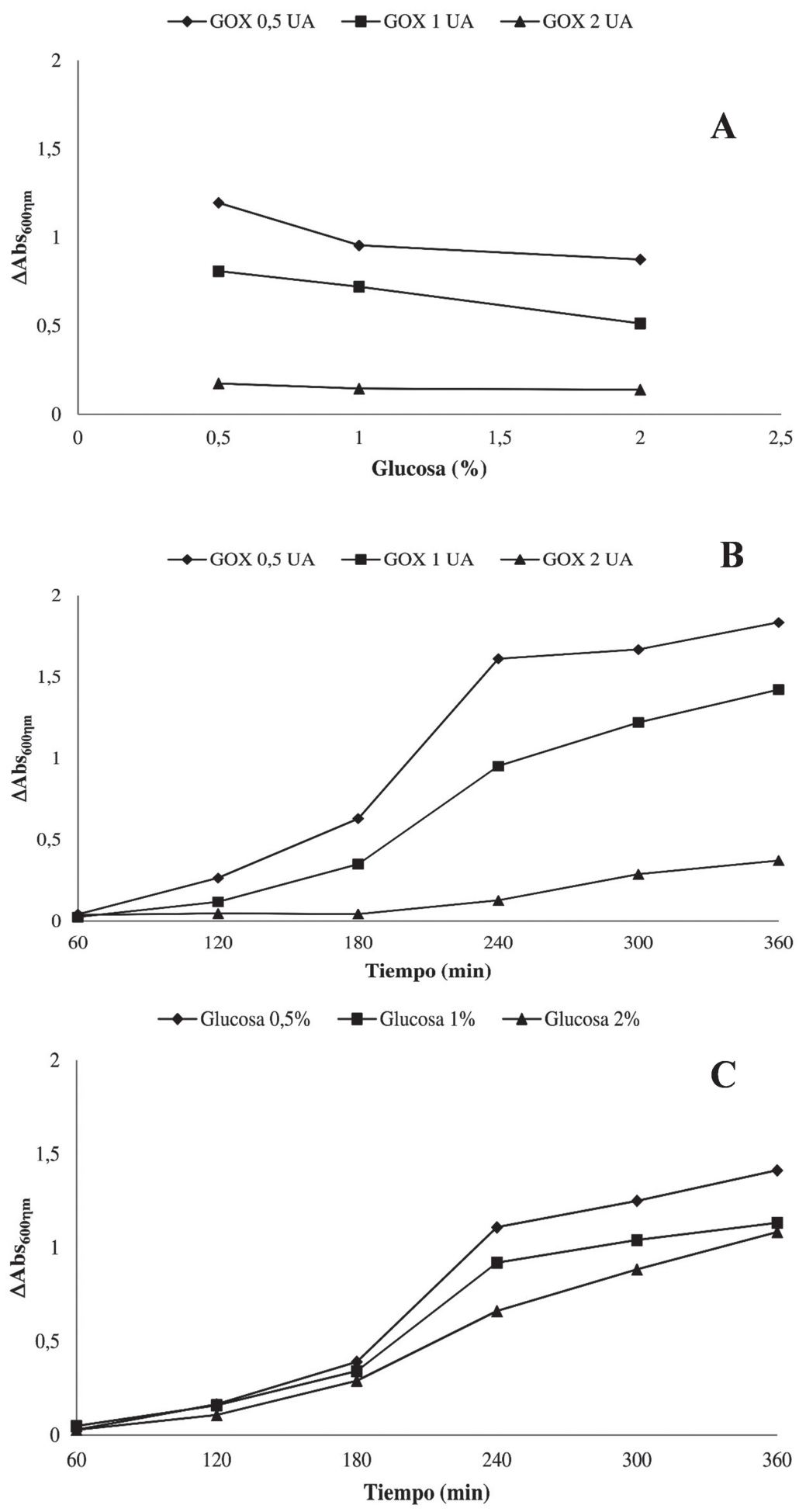

Figura 2. Interacciones entre los factores estudiados y el tiempo y su efecto sobre el crecimiento de S. tiphymurium: (A) GOX-Glucosa, (B) GOX-Tiempo, (C) Glucosa-Tiempo. 
peróxido y de ácido glucónico; sin embargo, Tiina \& Sandholm (1989) señalan que concentraciones excesivamente altas de GOX pueden ocasionar que el efecto inhibitorio sea breve y se pierda en un corto período de tiempo, ya que el sustrato se agota muy rápido y cesa la producción del peróxido y el ácido.

Por otra parte, es importante destacar que los tratamientos T1, T2 y T3, correspondientes a las combinaciones de GOX $0,5 \mathrm{U} / \mathrm{mL}$ con diferentes niveles de glucosa, en determinados momentos del experimento, exhibieron valores de $\Delta$ Abs600nm, significativamente superiores al control, indicativo de una estimulación del crecimiento bacteriano. Específicamente, T1 (GOX 0,5U/mL y glucosa $0,5 \%$ ) provocó un incremento de la turbidez superior al control, desde los 180 minutos hasta el tiempo final del estudio, mientras que las combinaciones con 1 y $2 \%$ de glucosa (T2 y T3) exhibieron valores significativamente superiores al control, hasta los 240 minutos. Este hecho permite inferir que, a bajas concentraciones de la enzima, parte de la glucosa adicionada al medio puede ser utilizada por el microorganismo como fuente de carbono para sus funciones metabólicas, estimulando el crecimiento bacteriano.

Adicionalmente, se consideraron 3 contrastes ortogonales o comparaciones particulares de promedios de tratamientos () de interés, a fin de analizar, con más detalle, las diferencias encontradas; los contrastes considerados fueron:

i. Contraste 1. Niveles promedios de GOX 2 UA contra el triple del promedio del control

ii. Contraste 2. Promedio de GOX 2 -Glucosa 0,5 contra el promedio de GOX 2-Glucosa 2

iii. Contraste 3. Promedio de GOX 2-Glucosa 2 contra el promedio de GOX 2-Glucosa 1

Los resultados de la prueba de Scheffé aplicada sobre los contrastes indican que, a partir de la segunda hora, se evidenció que el efecto de agregar $2 \mathrm{U} / \mathrm{mL}$ de GOX inhibió significativamente el crecimiento de $S$. typhimurium, con respecto al control. Asimismo, los contrastes 2 y 3 muestran que los efectos de agregar 0,5 y $1 \%$ glucosa con $2 \mathrm{U} / \mathrm{mL}$ de GOX son estadísticamente iguales con glucosa al $2 \%$, es decir, la contribución de agregar glucosa no es tan importante como agregar la enzima, a razón de $2 \mathrm{U} / \mathrm{mL}$. Confirmando lo observado por la interacción entre estos factores, es decir, que para $2 \mathrm{U} / \mathrm{mL}$ de GOX el efecto sobre el crecimiento es similar, si se adicionan $0,5,1$ y $2 \%$ de glucosa, por lo que se puede trabajar con la menor proporción de glucosa y ocasionar el efecto inhibitorio (Tabla 2).

Tabla 2. Contrastes ortogonales y prueba de Scheffé clasificados por tiempo: A) S. typhimurrium; B) S. enteriditis.

\begin{tabular}{|l|c|c|c|c|c|c|}
\hline \multirow{2}{*}{ Contrastes } & \multicolumn{7}{|c|}{ S. typhimurrium } \\
\cline { 2 - 7 } & $\mathbf{6 0}$ & $\mathbf{1 2 0}$ & $\mathbf{1 8 0}$ & $\mathbf{2 4 0}$ & $\mathbf{3 0 0}$ & $\mathbf{3 6 0}$ \\
\hline Contraste 1 & 0,0053 & $-0,2187$ & $-0,5294$ & $-1,0308$ & $-1,2035$ & $-1,6378$ \\
\hline$p$-valor & 1,0000 & $<0,0001^{*}$ & $<0,0001^{*}$ & $<0,0001^{*}$ & $<0,0001^{*}$ & $<0,0001^{*}$ \\
\hline Contraste 2 & 0,0059 & 0,0086 & 0,0194 & 0,0801 & 0,0613 & 0,0345 \\
\hline$p$-valor & 1,0000 & 1,0000 & 1,0000 & 0,9552 & 0,9996 & 1,0000 \\
\hline Contraste 3 & 0,0766 & 0,0439 & 0,0161 & 0,0037 & 0,0512 & $-0,1430$ \\
\hline$p$-valor & 0,1134 & 0,9167 & 1,0000 & 1,0000 & 0,9999 & 0,9727 \\
\hline & \multicolumn{7}{|c|}{ S. enteriditis } \\
\hline \multirow{2}{*}{ Contrastes } & \multicolumn{7}{|c|}{ Tiempo (min) } & \\
\cline { 2 - 8 } & $\mathbf{6 0}$ & $\mathbf{1 2 0}$ & $\mathbf{1 8 0}$ & $\mathbf{2 4 0}$ & $\mathbf{3 0 0}$ & 360 \\
\hline Contraste 1 & $-0,0264$ & $-0,1969$ & $-0,4517$ & $-0,2561$ & $-0,4312$ & $-0,5526$ \\
\hline$p$-valor & 0,7074 & 0,0741 & 0,2121 & 0,7928 & $0,0363 *$ & $0,0057^{*}$ \\
\hline Contraste 2 & 0,0119 & 0,0125 & 0,0384 & $-0,0346$ & $-0,0709$ & 0,0611 \\
\hline$p$-valor & 0,9995 & 1,0000 & 1,0000 & 1,0000 & 1,0000 & 1,0000 \\
\hline Contraste 3 & 0,0127 & 0,0049 & $-0,0050$ & $-0,0072$ & $-0,1402$ & $-0,1203$ \\
\hline$p$-valor & 0,9992 & 1,0000 & 1,0000 & 1,0000 & 0,9937 & 0,9986 \\
\hline
\end{tabular}

Nota: (*) Diferencia o efecto estadísticamente significativo al 5\%. 
El análisis permitió demostrar, que el crecimiento de $S$. typhimurium se ve afectado por la adición de GOX y glucosa al medio y que la magnitud de dicho efecto depende de las concentraciones empleadas, por lo que la inhibición ocasionada es dosis-dependiente, tal como lo han demostrado estudios previos (Tiina \& Sandholm, 1989; Yoo \& Rand, 1995; Massa et al. 2001; Noguera et al. 2014).

\section{B) S. enteritidis}

En la figura $1 \mathrm{~B}$, se puede apreciar que las distintas combinaciones de GOX y de glucosa afectaron diferencialmente el crecimiento de $S$. enteritidis en el tiempo. De manera general, se observó que los tratamientos con GOX de 0,5 y $1 \mathrm{U} / \mathrm{mL}$ exhibieron, en la mayoría de los tiempos evaluados, valores de $\Delta$ Abs600nm, superiores al control, mientras que los correspondientes a la concentración de 2 GOX, estuvieron por debajo.

La prueba de Mauchly indicó que la matriz de varianzas y de covarianzas no fue esférica $(p<0,001)$, por lo cual, se utilizaron los $p$-valores ajustados para realizar el ANOVA, que dejó ver el efecto de la enzima GOX ( $F=103,69 ; 2$ gdl; $p<0,001)$ sobre el crecimiento, pero no hubo efecto de la glucosa ( $\mathrm{F}=0,44 ; 2 \mathrm{gdl} ; p=0,650)$, ni interacción GOX-Glucosa $(\mathrm{F}=0,24 ; 4 \mathrm{gdl} ; p=0,31)$, comportamiento distinto al observado para $S$. tiphymurium.

Por otra parte, se confirmó el efecto del factor tiempo $(\mathrm{F}=535,49$; 5gdl; $p<0,001)$ y de la interacción GOX-Tiempo $(\mathrm{F}=15,24 ; 10 \mathrm{gdl} ; p<0,001)$, pero no hubo efecto de las interacciones Glucosa-Tiempo ( $\mathrm{F}=0,99 ; 10 \mathrm{gdl} ; p=0,457)$ ni GOX-Glucosa-Tiempo ( $\mathrm{F}=0,014 ; 20 \mathrm{gdl} ; p=0,528)$. Estos resultados indican que el crecimiento de $S$. enteritidis fue afectado solo por los niveles de GOX, utilizados durante el tiempo de estudio.

Con respecto a los efectos principales de GOX, la prueba de comparaciones múltiples de Tukey para GOX, indicó que el mayor crecimiento ocurrió con el nivel más bajo de GOX $0,5 \mathrm{U} / \mathrm{mL}\left(=0,980^{\mathrm{A}}\right)$, seguido de $1 \mathrm{U} / \mathrm{mL}\left(=0,836^{\mathrm{B}}\right)$ y el menor crecimiento, se obtuvo con $2 \mathrm{U} / \mathrm{mL}$ de GOX $\left(=0,483^{\mathrm{C}}\right)$. Las letras en los superíndices representan los grupos de medias de Tukey, que presentan diferencias significativas; sin embargo, el efecto fue menor al observado en el caso del serotipo S. typhimurium.

Para la interacción GOX $\times$ Tiempo, promediada sobre los niveles de glucosa, la respuesta fue homogénea a los 60 minutos y mostró una clara diferenciación desde los 120 minutos. A partir de ese momento, el crecimiento resultó menor, en la medida que aumentó el nivel de GOX, de tal forma, que las menores respuestas de $\Delta$ Abs600nm siempre las exhibió la concentración $2 \mathrm{U} / \mathrm{mL}$ de GOX, mientras que los niveles GOX 0,5 y $1 U / \mathrm{mL}$, tuvieron un comportamiento parecido entre sí (Figura 3). Las demás interacciones no se graficaron, por no resultar estadísticamente significativas.

Los resultados del ANOVA, a través del tiempo y la prueba de comparación de tratamientos contra control de Dunnett, demostraron que, para la primera hora, no hubo diferencias con respecto al control y fue a partir de la segunda hora (120 minutos), que se observaron diferencias significativas $(\mathrm{p}<0,001)$; a partir de ese instante, los tratamientos T7, T8 y T9 exhibieron los menores promedios.

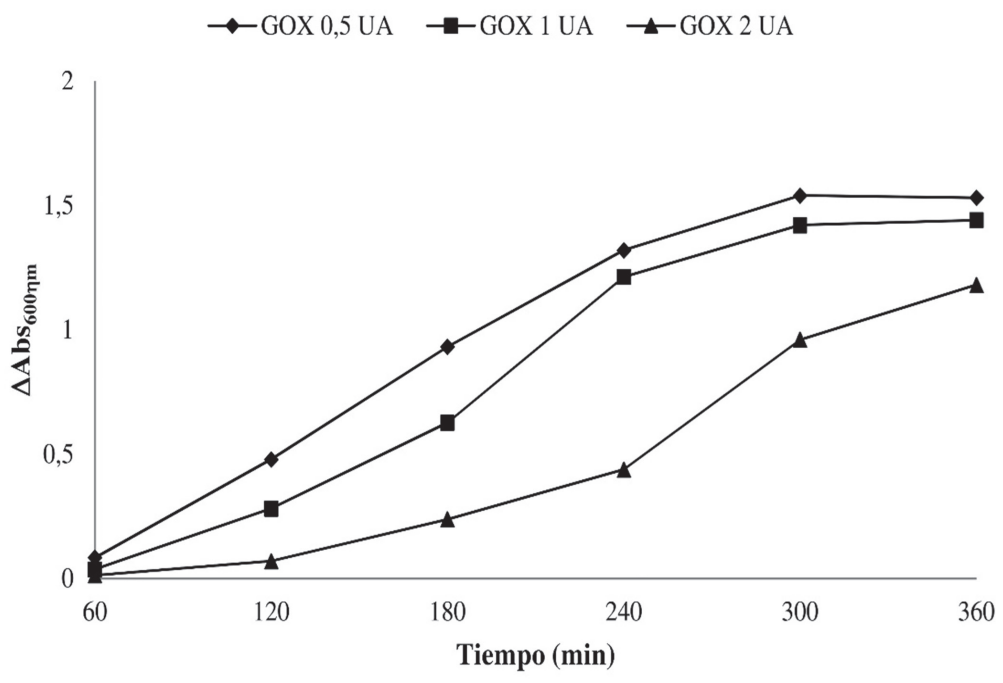

Figura 3. Interacción GOX $\times$ Tiempo y su efecto sobre el crecimiento de $S$. enteritidis. 
Al igual que para S. tiphymurium, se consideraron los mismos contrastes ortogonales, definidos anteriormente. Los resultados de la prueba de Scheffé evidenciaron que el efecto inhibitorio de agregar GOX $2 \mathrm{U} / \mathrm{mL}$ deviene estadísticamente significativo, a partir de las 5 horas (300 minutos). Adicionalmente, la ausencia de resultados estadísticamente significativos para el resto de los contrastes, ratifican la ausencia de efecto de la adición de glucosa, en sus diferentes niveles (Tabla 2B).

Estos hechos confirman que este serotipo resultó ser más tolerante a la acción del sistema GOX/glucosa, por lo que, probablemente, se requiere una dosis más elevada de ambos componentes, de enzima y del sustrato.

Estudios previos han descrito la tolerancia de algunas cepas pertenecientes a este género, a los productos de reacción de esta enzima. Frels et al. (1984) evaluaron la efectividad de un sistema compuesto de GOX, catalasa y dextrosa al $4 \%$, para retrasar el crecimiento de tres serotipos de Salmonella (S. heidelberg, S. enteritidis, S. typhimurium) presentes en los muslos de aves, y no encontraron un efecto significativo, quizás por la presencia de catalasa, que descompone el peróxido de hidrógeno. En la investigación realizada por Massa et al. (2001), con S. derby, concluyeron que solo elevadas concentraciones de GOX y glucosa podían ocasionar inhibición sobre el crecimiento de este serotipo.

En consecuencia, se puede concluir que el sistema GOX/ glucosa tiene potencial como aditivo para mejorar la calidad microbiológica de productos avícolas, ya que es capaz de inhibir el crecimiento de bacterias del género Salmonella aisladas de aves; sin embargo, se requieren de más estudios para optimizar las concentraciones y lograr la máxima inhibición frente a un grupo más amplio de especies bacterianas, asociadas al deterioro de este tipo de alimentos.

Agradecimientos: A la Universidad de Carabobo y a su Consejo de Desarrollo Científico y Humanístico (CDCH-UC), por el apoyo institucional y financiero, para desarrollar esta investigación. También, al Instituto Nacional de Investigaciones Agrícolas (INIA), por su apoyo institucional, para la realización de esta investigación. Financiación: Este proyecto fue financiado por el Consejo de Desarrollo Científico y Humanístico de la Universidad de Carabobo (CDCH-UC). Conflicto de intereses: El manuscrito fue preparado y revisado con la participación de todos los autores, quienes declaramos que no existe conflicto de intereses, que ponga en riesgo la validez de los resultados presentados.

\section{BIBLIOGRAFÍA}

1. BANKAR, S.B.; MAHESH, B.; SINGHAL, R.; ANANTHANARAYAN, L. 2009. Glucose oxidase - an overview. Biotechnology Advances. 27(4):489-501.
2. BERISTAIN-BAUZA, S.C.; PALOU, E.; LÓPEZ-MALO, C. 2012. Bacteriocinas: antimicrobianos naturales y su aplicación en los alimentos. Rev. Temas Selectos de Ingeniería de Alimentos. 6(2):64-78.

3. CAMACHO, O.; ACEDO, L.; MORENO, G.; SÁNCHEZ, R.; CASTILLÓN, L.; NAVARRO, M. 2010. Detección de Salmonella resistente a los antibióticos en vísceras de pollo. Rev. Biotecnia. XII(1):3-11.

4. CASTAÑEDA, M.; BRAÑA, D.; ROSARIO, C.; MARTÍNEZ, W. 2013. Calidad microbiológica de la carne de pollo. Libro Técnico 9, de la Facultad de Medicina y Zootecnia, de la Universidad Autónoma de México. 90p.

5. COMISIÓN VENEZOLANA DE NORMAS INDUSTRIALES -COVENIN-. Norma No 1291. 1988. Alimentos. Aislamiento e identificación de Salmonella. Disponible desde Internet en: http://portal.sencamer.gob.ve (con acceso 13/06/2017).

6. FRELS, J.M.; SAMUELSON, K.J.; FRONING, G.W.; RUPNOW, J.H. 1984. Evaluation of glucose oxidase-catalase treatment to improve the microbiological quality of poultry meat. Poultry Science. 63(4):841-843.

7. GARBOZA, F.; FRONTADO, R.; NOGUERA, N.; ÁVILA, H.; OJEDA, L.; RAMÍREZ, N.; TRIANA, J.; TRIANA, F. 2011. Uso de medios alternativos a base de hidrolizado de caseína y extracto de Aspergillus niger y su efecto sobre la expresión genética de una cepa de Escherichia coli. Rev. Soc. Venezolana de Microbiología. 31:138-143

8. GRACIANO-CRISTÓBAL, M.; SUMAYA-MARTÍNEZ, M.; BALOIS-MORALES, R.; RODRÍGUEZ-CARPENA, J.; JIMÉNEZ-RUIZ, E.; BAUTISTA-ROSALES, P.; MADRIGAL-SANTILLÁN, E. 2017. Efecto antimicrobiano de aditivos naturales en carne de cerdo cruda. Rev. Acta Agrícola y Pecuaria. 3(2):32-40.

9. HOLZAPFEL, W.; GEISEN, R.; SCHILLINGER, U. 1995. Biological preservation of foods with reference to protective cultures, bacteriocins and food-grade enzymes. Internal J. Food Microbiology. 24:343-362.

10. KAUFFMANN, F. 1966. The bacteriology of Enterobacteriaceae. The Williams \& Wilkins Company, Baltimore. 400p.

11. MASSA, S.; PETRUCCIOLI, M.; BROCCHI, G.; ATIERI, C.; SINIGAGLIA, M.; SPANO, G. 2001. Growth inhibition by glucose oxidase system of enterotoxic Esche- 
richia coli and Salmonella derby: in vitro studies. World J. Microbiology \& Biotechnology. 17(3):287291.

12. MERCADO, M.; ÁVILA J.; REY, M.; MONTOYA, M.; GAMBOA, A.; CARRASCAL, A.; CORREA, D. 2012. Brotes por Salmonella spp., Staphylococcus aureus y Listeria monocytogenes asociados al consumo de pollo. Revista Biomédica. 32:375-285.

13. MOLINA, N.; MILLÁN, B.; ARAQUE, M. 2010. Indicadores de calidad sanitaria y fenotipificación de Salmonella enterica aislada de pollo crudo comercializado en el área urbana de Mérida, Venezuela. Infectio. 14(3):174-185.

14. NILLIAN, E.; CHING, C.; FUNG, P.; ROBIN, T.; ANYI, U.; ZAINAZOR, T.; CHILEK, T.; RADU, S.; NISHIBUCHI, M. 2011. Simultaneous detection of Salmonella spp., Salmonella enteritidis and Salmonella typhimurium in raw salad vegetables and vegetarian burger patties. Food and Nutrition Sciences. 2(10):1077-1081.

15. NOGUERA, N.; HERNÁNDEZ, A.; JIMÉNEZ, A.; REQUENA, D.; RAMÍREZ, N.; OJEDA, L. 2014. Efecto del sistema glucosa oxidasa/glucosa sobre el crecimiento de Escherichia coli ATCC 25922 en leche. Rev. Venezolana Ciencia y Tecnología Alimentos). 5(1): 057-069.

16. PANIAGUA-CONTRERAS, G.; MONROY-PÉREZ, E.; VACA-PACHECO, S. 2008. Prevalencia de Salmonella spp. en diarrea de niños de una zona urbana del Estado de México. Rev. Médica del Hospital General de México. SS 71(4):192-198.

17. RINCÓN, D.; RAMÍREZ, R.; VARGAS, J. 2011. Transmisión de Salmonella enterica a través de huevos de gallina y su importancia en salud pública. Salud UIS. 43(2):167-177.

18. TIINA, M.; SANDHOLM, M. 1989. Antibacterial effect of the glucose oxidase-glucose system on food-poisoning organisms. Internal J. of Food Microbiology. 8:165-174.
19. URIBE, C.; SUÁREZ, M. 2006. Salmonelosis no tifoidea y su transmisión a través de alimentos de origen aviar. Colombia Médica. 37(2):151-158.

20. VÁSQUEZ, S.; SUÁREZ, H.; ZAPATA, S. 2009. Utilización de sustancias antimicrobianas producidas por bacterias acido lácticas en la conservación de la carne. Rev. Chilena de Nutrición. 36(1):64-71.

21. WATMAN, D.; GAST, R.; MALLISON, E. 2008. Salmonellosis In: A laboratory manual for the insolation and identification of avian pathogens. Published by the American Association of Avian Pathologist, $5^{\text {th }}$ ed. p.3-9.

22. WHO. 2015. World Health Organization. Inocuidad de los alimentos. Nota descriptiva $N^{\circ} 399$. Disponible desde Internet en: http://www.who.int/mediacentre/ factsheets/fs399/es/ (con acceso:27/09/2016).

23. WHO. 2016. World Health Organization. Salmonella (no tifoidea). Nota descriptiva. Disponible desde Internet en: http://www.who.int/mediacentre/factsheets/ fs $139 /$ es/ (con acceso 24/02/2017).

24. YANG, Z. 2000. Antimicrobial compounds and extracellular polysaccharides produced by lactic acid bacteria: structures and properties. University of Helsinki Department of Food Technology: Helsinki, Finland, $61 \mathrm{p}$.

24. YOO, W.; RAND, A.G. 1995. Antibacterial effect of glucose oxidase on growth of Pseudomonas fragi as related to $\mathrm{pH}$. J. Food Science. 60(4):868-871.

26. ZOGHBI, N.; OJEDA, L.; NOGUERA, N.; YÉPEZ, A.; CAMARGO, H.; TRIANA-ALONSO, F. 2008. Extracción y purificación de glucosa oxidasa para fines diagnósticos producida en medios a base de fertilizantes y azúcar industrial. Rev. Soc. Venezolana de Microbiología. 28(1):31-37.

Recibido: Julio 2 de 2017

Aceptado: Mayo 5 de 2018

Cómo citar:

Noguera-Machado, N.C.; Ojeda-Ojeda, L.E.; Pérez-Ybarra, L.; Brett, M.; García, K.; Yépez, A.; Triana, J.L. 2018. Efecto de la combinación de glucosa oxidasa/glucosa sobre el crecimiento de bacterias del género Salmonella aisladas de aves de corral. Rev. U.D.C.A Act. \& Div. Cient. 21(1): 127-136. 